Support Centre, Tallinn, Estonia; 'Latvia's Association for Family Planning and Sexual Health, Riga, Latvia; ${ }^{5}$ Aids Hilfe Potsdam, Potsdam, Germany; ${ }^{6}$ C.A. Prima, Bratislava, Slovakia; ${ }^{7}$ Health and Social Development Foundation, Sofia, Bulgaria; ${ }^{8}$ Romanian Associations against AIDS, Bucharest, Romania

Background Prostitution is a frequent and often controversial discussion topic at expert and political levels in Europe. Still evidencebased knowledge of sex work in general, and the situation of the SWs in particular, is scarce. BORDERNETwork addressed these gaps with an integrated bio-behavioural survey in seven EU countries. It compiled contextualised knowledge on the health and social situations of SWs, detected prevalence of and vulnerability to HIV/STIs, and formulated prevention practise recommendations.

Methods Behavioural and epidemiological data was collected between March 2011 and February 2012 through qualitative faceto-face interviews (an 85-item questionnaire) and blood screening tests (HIV, Syphilis, HCV, and HBV) among 956 SWs (respondentsand service-driven sample) in six capital cities and a border area.

Results The findings outlined multiple risks: About 38\% of the SWs had experience injecting drugs, $60 \%$ lacked health insurance. $59 \%$ had had an HIV test in the past year, but STI/sexual health services are hardly utilised. $77.1 \%$ had not attended an STI-specialist and $51.1 \%$ had not visited a gynaecologist in the past year. The prevalence for HIV was $4.6 \%$, for Syphilis - $4.5 \%$, for Hepatitis B $6.2 \%$, and for Hepatitis C - $24 \%$ (over $90 \%$ among PWID). Risk predictors linked in particular to increased prevalence of Syphilis, HBV, and HCV were drug and alcohol use before/during sex work and inconsistent condom use during oral/vaginal sex with clients.

Conclusions The behavioural and social determinants of risk, including precarious living conditions and stigma, multiply the vulnerability of SWs impeding their access to health and social care. To that end policy regulations should endorse the creation of structures for early and easy access to health services. An adequate health care provision package (incl. sexual health) should be envisaged including those SWs lacking health, social insurance and legal status in the country of stay also.

\section{P3.306 POPULATION CHANGE AND MALE CLIENTS OF SEX WORK IN U.S. METROPOLITAN AREAS}

doi:10.1136/sextrans-2013-051184.0760

M A Villarroel. Johns Hopkins Bloomberg School of Public Health, Baltimore, MD, United States

Background Rate of population change gives an indication of the average economic prosperity in a geographic area that reflects changing conditions to its physical and social environment which may influence the STIs risk behaviours found. Men who buy sex from sex workers (clients) are at high-risk of STIs and key in the spread infection to the general population. This study analyses census data matched with survey data to examine the association between population change in U.S. metropolitan counties with clients.

Methods A sample of 385 metropolitan men embedded in a 19992000 national probability telephone survey was linked to 1990 and 2000 U.S. Census county population counts. County population change between 1990 and 2000 was measured categorically and as a transformed continuous variable to account for negative growth and its skewed distribution. Poisson regression was used to obtain prevalence ratio (PR).

Results The sample was $73 \%$ non-Hispanic white, median age of 36 years, $77 \%$ were currently married or living with a partner and $39 \%$ had completed at least four years of college. The prevalence of clients was $14.8 \%$ (CI $11.5-18.9 \%$ ), differing significantly by the population relative change of the county of residence $(\mathrm{P}<0.05)$. The lowest and highest prevalence of clients was observed among men residing in counties that loss population $(6.0 \%$ (CI, 2.0-17.2) and grew rapidly above the national average $(21.4 \%$, CI, $13.5 \%-32.3 \%)$.
Using the transformed continuous measure for population change, there was a statistically significant increase in the ratio of male clients with a unit increase in population growth above the national average (unadjusted PR, 0.2, CI, 0.1-0.5, P < 0.010).

Conclusion The relationship between rate of urbanisation in metropolitan areas and male clients provides support for demographic changes influencing level of STI risks in the population and may contribute to development of comprehensive STI prevention activities.

\section{P3.307 POPULATION GROWTH WITHIN REGIONAL PATTERN OF POPULATION CHANGE AND MALE CLIENTS OF SEX WORKERS IN U.S. METROPOLITAN AREAS}

doi:10.1136/sextrans-2013-051184.0761

M A Villarroel. Johns Hopkins Bloomberg School of Public Health, Baltimore, MD, United States

Background Male clients of sex workers are at high-risk of HIV and are a key group in the spread of sexually transmitted infections to the general population. Places undergoing population growth are altering their economic, physical and social environments which may influence the pattern of sexual risk behaviour. However population growth is not uniform within or between U.S. metropolitan areas and there is an interrelated pattern of growth within a metropolitan area. This study examined the association of county population growth that was slower versus similar and faster than its own metropolitan area's pace of population change with male clients in a sample of men $(N=303)$ residing in U.S. metropolitan areas embedded in a 1999-2000 national probability telephone survey of STD behavioural measurements.

Methods Population counts for non-institutionalised population at the county level were obtained from the 1990 and 2000 U.S. Census and the total household population in each metropolitan area was calculated by adding the household population of all its component counties. County population change relative to its metropolitan area was quantified using location quotient. Poisson regression was used to obtain prevalence ratio (PR).

Results The prevalence of male clients was $17.5 \%$. In unadjusted analysis, there was no statistically significant relationship between clients and whether the county of residence experienced a rate of growth that was slower versus similar ( $\mathrm{PR}=1.79,95 \% \mathrm{CI} 0.9-3.3, \mathrm{P}>0.05)$ or faster ( $\mathrm{PR}=1.4,95 \%$ CI $0.7-2.9, \mathrm{P}>0.05)$ to its overall metropolitan area. After adjusting for county-level demographical differences at the starting period, clients were however more likely to reside in counties that experienced a similar ( $\mathrm{PR}=2.33,95 \% \mathrm{CI} 1.1-5.0, \mathrm{P}<0.05)$ or a faster $(\mathrm{PR}=2.5,95 \% \mathrm{CI} 1.1-5.9, \mathrm{P}<0.05)$ pace of growth.

Discussion Male clients of sex workers may not be similarly distributed across metropolitan areas and demographic changes may influence patterns of STI risks.

\section{P3.308 THE CHANGING MALE SEX WORKER POPULATION IN LONDON (2002 - 2012)}

doi:10.1136/sextrans-2013-051184.0762

R Malek, L Mulka, G King, A Scott, D Wilkinson. Imperial College Healthcare NHS Trust, London, UK

Background With freedom of movement across European borders, increasing globalisation and emergence of new major economies, the UK has seen significant changes to the composition of nationalities migrating to the UK over the last ten years. In turn this has changed the working population of the UK. The objective of this study was to investigate the changes in nationalities of male sex workers (MSW) attending a dedicated clinic for MSW in London over the last decade.

Methods Clinic records for MSW attending a dedicated clinic in Central London were reviewed (1/1/2002 - 31/12/2002 and 